\title{
Predictive Role of Temporal Changes in Intratumoral Metabolic Heterogeneity During Palliative Chemotherapy in Patients with Advanced Pancreatic Cancer: A Prospective Cohort Study
}

\author{
Shin Hye Yoo*1, Seo Young Kang*2, Gi Jeong Cheon ${ }^{2}$, Do-Youn $\mathrm{Oh}^{1,3}$, and Yung-Jue Bang ${ }^{1,3}$ \\ ${ }^{I}$ Department of Internal Medicine, Seoul National University Hospital, Seoul, Korea; ${ }^{2}$ Department of Nuclear Medicine, Seoul National \\ University Hospital, Seoul, Korea; and ${ }^{3}$ Cancer Research Institute, Seoul National University College of Medicine, Seoul, Korea
}

\begin{abstract}
Metabolic intratumoral heterogeneity (ITH) is known to be related to cancer treatment outcome. However, information on the temporal changes in metabolic ITH during chemotherapy and the correlations between metabolic changes and treatment outcomes in patients with pancreatic cancer is sparse. We aimed to analyze the temporal changes in metabolic ITH and the predictive role of its changes in advanced pancreatic cancer patients who underwent palliative chemotherapy. Methods: We prospectively enrolled patients with unresectable locally advanced or metastatic pancreatic cancer before first-line palliative chemotherapy. ${ }^{18} \mathrm{~F}$-FDG PET was performed at baseline and at the first response follow-up. SUVs, volumetric parameters, and textural features of the primary pancreatic tumor were analyzed. Relationships between the parameters at baseline and first follow-up were assessed, as well as changes in the parameters with treatment response, progression-free survival (PFS), and overall survival (OS). Results: Among 63 enrolled patients, the best objective response rate was $25.8 \%$ (95\% confidence interval [Cl], $14.6 \%-37.0 \%)$. The median PFS and OS were $7.1 \mathrm{mo}(95 \% \mathrm{Cl}, 5.1-$ $9.7 \mathrm{mo})$ and $10.1 \mathrm{mo}(95 \% \mathrm{Cl}, 8.6-12.7 \mathrm{mo})$, respectively. Most parameters changed significantly during the first-line chemotherapy, in a way of reducing ITH. Metabolic ITH was more profoundly reduced in responders than in nonresponders. Multiple Cox regression analysis identified high baseline compacity $(P=0.023)$ and smaller decreases in $\mathrm{SUV}_{\text {peak }}(P=0.007)$ and entropy gray-level cooccurrence matrix $(P=0.033)$ to be independently associated with poor PFS. Patients with a high carbohydrate antigen 19-9 $(P=0.042)$, high pretreatment $\operatorname{SUV}_{\text {peak }}(P=0.008)$, and high coefficient of variance at first follow-up $(P=0.04)$ showed worse OS. Conclusion: Reduction in metabolic ITH during palliative chemotherapy in advanced pancreatic cancer patients is associated with treatment response and might be predictive of PFS and OS.
\end{abstract}

Key Words: ${ }^{18} \mathrm{~F}-\mathrm{FDG}$ PET; intratumoral heterogeneity; tumor metabolism; pancreatic cancer; texture analysis

J Nucl Med 2020; 61:33-39

DOI: 10.2967/jnumed.119.226407

Received Jan. 23, 2019; revision accepted Jun. 3, 2019.

For correspondence or reprints contact: Do-Youn Oh, Department of Internal Medicine, Seoul National University Hospital, 101 Daehak-ro, Jongno-gu, Seoul 03080, Korea.

E-mail: ohdoyoun@snu.ac.kr

${ }^{*}$ Contributed equally to this work.

Published online Jun. 14, 2019.

COPYRIGHT (C 2020 by the Society of Nuclear Medicine and Molecular Imaging.
M different levels of cellular proliferation, invasivenes, metastatic potential, and susceptibility to anticancer drugs and show complicated cell-cell interactions. This intratumoral heterogeneity (ITH) has been found to be clinically important with regard to patient outcome and response to therapy, resulting in growing interest in this area of research. ITH is most commonly thought to be the result of subclonal genetic diversity, which leads to variations in stromal architecture, oxygen consumption, and glucose metabolism (1).

To evaluate ITH in a noninvasive manner, various imaging modalities can be used for depicting ITH by combining data from 3 -dimensional elements called voxels $(1,2)$. CT or MRI has benefits to aid diagnosis, characterization, and response assessment of tumors using ITH. ${ }^{18} \mathrm{~F}-\mathrm{FDG}$ PET/CT, which quantifies tumor metabolic activity, is also a useful imaging modality for diagnosis, follow-up assessment, and response to treatment. With regard to regional variations in glucose metabolism, ITH can be evaluated by ${ }^{18} \mathrm{~F}$-FDG PET/CT. Studies on metabolic ITH suggest that it might affect the outcomes and treatment responses of patients with several types of malignancies (3-6).

Patients with unresectable or metastatic pancreatic cancer have a major disease burden and show high mortality and poor response to treatment (7). Pancreatic cancer is a stroma-rich tumor that usually shows an elevated level of ITH (8). Because obtaining sufficient tissue for identifying ITH histopathologically is usually difficult, evaluating metabolic ITH in pancreatic cancer patients with imaging modalities is a reasonable alternative. There are a few retrospective studies (9-11) that have investigated the predictive role of metabolic ITH in pancreatic cancer. However, the studies address heterogeneous patient groups, raising questions about the reliability of the results. In addition, whether a change in metabolic ITH after chemotherapy is associated with the treatment response and outcome of patients remains unclear.

We aimed to analyze the temporal changes in metabolic ITH and the predictive role of $\Delta$-radiomics in a prospective cohort of patients with unresectable or metastatic pancreatic cancer who underwent palliative chemotherapy.

\section{MATERIALS AND METHODS}

\section{Patients and Data Collection}

We prospectively enrolled patients with advanced gastric cancer, pancreatic cancer, or biliary tract cancer beginning in October 2013. The inclusion criteria were as follows: histopathologically confirmed 


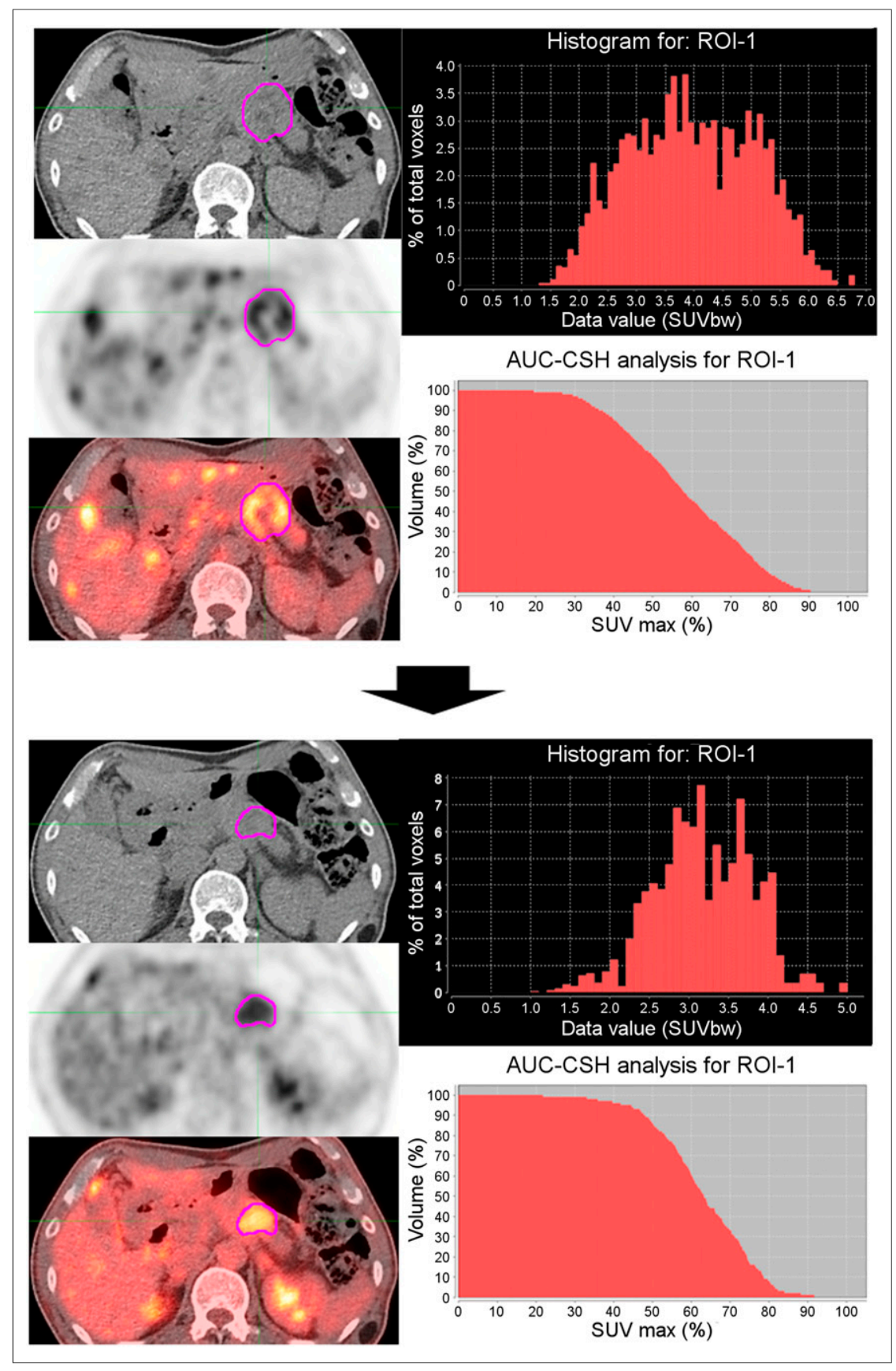

FIGURE 1. Representative image showing metabolic ITH changes from ${ }^{18} \mathrm{~F}-\mathrm{FDG}$ PET/CT images at T1 (top) and T2 (bottom) in 61-y-old man. Histogram and AUC-CSH show decreased metabolic ITH after palliative chemotherapy. AUC-CSH = area under curve of cumulative SUV volume histogram; $\mathrm{ROI}=$ region of interest.

advanced cancer; unresectable, locally advanced, or metastatic cancer; planned palliative chemotherapy (chemotherapy-naïve); and written informed consent. For this analysis, we included pancreatic cancer patients only. Cases of primary pancreatic tumor that could not be examined were excluded (Supplemental Figure 1; supplemental materials are available at http://jnm.snmjournals.org). Demographic and clinical information including carcinoembryonic antigen level and carbohydrate antigen 19-9 (CA 19-9) level was collected. Response to chemotherapy was assessed by the RECIST guideline, version 1.1 (12), which was applied to both primary and metastatic lesions. ${ }^{18} \mathrm{~F}-\mathrm{FDG}$ PET/CT was performed before the initiation of palliative chemotherapy (T1) and at the time of the first evaluation of response (T2), usually after 2 or 3 cycles of chemotherapy, for all participants and then serially performed for each evaluation of response, when possible. The study protocol was reviewed and approved by the institutional review board of the Seoul National University Hospital (approval H1307-132-508). We conducted the study in accordance with the principles of the Declaration of Helsinki.

\section{F-FDG PET/CT Protocol}

All patients were administered ${ }^{18} \mathrm{~F}-\mathrm{FDG}$, $5.18 \mathrm{MBq} / \mathrm{kg}$, intravenously after at least $6 \mathrm{~h}$ of fasting. Serum glucose levels were less than $150 \mathrm{mg} / \mathrm{dL}$ at the time of ${ }^{18} \mathrm{~F}-\mathrm{FDG}$ administration. ${ }^{18} \mathrm{~F}-\mathrm{FDG} \mathrm{PET} / \mathrm{CT}$ using a dedicated scanner (Biograph 40 TruePoint; Siemens) was performed at $60 \mathrm{~min}$ after the injection. After a low-dose CT scan for attenuation correction $(120 \mathrm{kV}, 3.75-\mathrm{mm}$ slice thickness), a consecutive emission scan was acquired in 3 dimensions (5-6 bed positions, $2.5 \mathrm{~min} / \mathrm{bed}$ position, 21.6-cm increments). PET images were reconstructed onto a matrix of $128 \times 128$ using 3-dimensional ordered-subsets expectation maximization (2 iterations, 21 subsets). Details on the ${ }^{18} \mathrm{~F}$ FDG PET/CT procedure have been described in our previous reports $(13,14)$.

\section{Analysis of SUV and Texture Analysis (TA)}

A nuclear medicine specialist performed tumor delineation, metabolic parameter analysis, and TA without knowledge of any clinical information. PET Edge, a gradientbased delineation tool in MIMVista (version 4.1; MIM Software Inc.), was used for tumor segmentation. The volume of interest (VOI) of the primary pancreatic lesion was automatically defined. Metastatic lesions were not used in the analysis. SUV parameters, including $\mathrm{SUV}_{\text {mean }}, \mathrm{SUV}_{\text {max }}$, and $\mathrm{SUV}_{\text {peak }}$, and volumetric parameters, including metabolic tumor volume and total lesion glycolysis, (TLG) were extracted from the VOI. The coefficient of variance $(\mathrm{CoV})$, which is defined as the $\mathrm{SD}$ of the SUVs divided by the $\mathrm{SUV}_{\text {mean }}$ was calculated from the extracted parameters. The CoV correlates positively with the degree of heterogeneity in the VOI (6).

For TA, ${ }^{18} \mathrm{~F}$-FDG PET/CT images and delineation data were imported to LifeX (version 4.0), which is a multiplatform and easy-to-use freeware program (15). Each ${ }^{18} \mathrm{~F}$-FDG PET/ CT image was resampled into a 64-level gray scale by a fixed-binwidth method with 0.3-SUV-unit scaling, from the minimum to an $\mathrm{SUV}_{\max }$ of $0-20$. We included the histogram indices and shape indices as first-order parameters, indices for the gray-level cooccurrence matrix (GLCM) as second-order parameters, and the neighboring graylevel dependence matrix as higher-order parameters (16). Definitions and explanations of the parameters derived from TA are described in Supplemental Table 1. Histogram indices provide information derived from global histogram analysis. GLCM takes into account the arrangements 
of pairs of voxels to extract textural indices. The neighboring gray-level dependence matrix corresponds to the difference in the gray level of a single voxel and its 26 neighbors in 3 dimensions. Figure 1 depicts a representative image of changes in metabolic ITH.

\section{Statistical Analysis}

On the basis of previous studies $(9,17)$, sample size calculation indicated that at least 61 participants were needed (95\% confidence interval [CI]; type II error rate of $20 \%$ ) to reach an assumed relative hazard of 2.5 and a censoring rate of $30 \%$, accounting for a drop-out rate of $5 \%-10 \%$ at $\mathrm{T} 2$.

Differences between the T1 and T2 parameters of SUV and TA were assessed by the paired $t$ test (for parametric analysis) or Wilcoxon signed rank test (for nonparametric analysis). According to the RECIST response to chemotherapy, we divided patients into 2 groups: responders (complete response or partial response) and nonresponders (stable disease or progressive disease). The Student $t$ test or Mann-Whitney test was used for comparing variables for responders with those for nonresponders, after testing for normality by the Shapiro-Wilks test.

The predictive performance of SUV and TA parameters was investigated by time-dependent receiver-operating characteristic curves for progression-free survival (PFS) and overall survival (OS). PFS was defined as the time from initiation of first-line chemotherapy to the date of disease progression or death. OS was calculated from the time of initiation of first-line chemotherapy to the date of death or last follow-up. The area under the receiver-operating characteristic curve (AUC) was calculated by the survivalROC package in $\mathrm{R}$ open-source statistical software (R Foundation). We dichotomized each variable as high or low, with different cutoffs that maximized the AUC. Univariate Cox proportional hazards regression analysis was performed to investigate the associations of clinical variables and of SUV and TA parameters with PFS and OS. Because of the large number of SUV and TA parameters, we included only variables with AUCs of more than 0.6 in the univariate analysis. After omission of the variables with multicollinearity, backward-selected multivariable Cox regression analyses were conducted for the significant variables, with a $P$ value of less than 0.05 , as identified by the univariate analysis. Survival estimates were determined by the Kaplan-Meier method and were compared by the log-rank test. Two-sided $P$ values of less than 0.05 were considered statistically significant. All statistical tests were 2-sided and were performed using STATA, version 12 (StataCorp LP), and R software.

\section{RESULTS}

\section{Patients and Baseline Characteristics}

With May 2018 as the cutoff date for data, 63 patients were enrolled in the study (Supplemental Fig. 1). For analyses comparing T1 and T2, we excluded 2 patients who did not undergo follow-up $\mathrm{PET} / \mathrm{CT}$ and 1 patient whose VOI at T2 could not be obtained.

\section{TABLE 1}

Comparison of Parameters Between Responders $(n=16 ; 25.8 \%)$ and Nonresponders $(n=46 ; 74.2 \%)$

\begin{tabular}{|c|c|c|c|c|c|c|c|c|c|}
\hline \multirow[b]{2}{*}{ Parameter } & \multicolumn{3}{|c|}{$\mathrm{T} 1(n=63)$} & \multicolumn{3}{|c|}{$\mathrm{T} 2(n=60)$} & \multicolumn{3}{|c|}{ Change $^{\star}(n=60)$} \\
\hline & Nonresponders & Responders & $P$ & Nonresponders & Responders & $P$ & Nonresponders & Responders & $P$ \\
\hline \multicolumn{10}{|c|}{ Conventional indices } \\
\hline $\mathrm{SUV}_{\max }$ & $8.51(0.51)$ & $9.13(0.81)$ & 0.52 & $6.44(2.72)$ & $6.09(2.75)$ & 0.739 & $-18.73(4.53)$ & $-31.94(5.85)$ & 0.128 \\
\hline SUV $_{\text {peak }}$ & $6.98(3.29)$ & $7.72(2.73)$ & 0.394 & $5.11(2.51)$ & $4.25(3.06)$ & 0.283 & $-22.89(31.23)$ & $-47.19(32.00)$ & 0.007 \\
\hline TLG & $158.90(204.55)$ & $231.05(226.52)$ & 0.085 & $95.92(134.86)$ & $66.51(68.97)$ & 0.318 & $-22.56(51.07)$ & $-72.19(20.52)$ & 0.0003 \\
\hline $\mathrm{CoV}$ & $0.27(0.14)$ & $0.28(0.07)$ & 0.204 & $0.23(0.11)$ & $0.20(0.06)$ & 0.447 & $-12.45(20.87)$ & $-26.62(22.02)$ & 0.029 \\
\hline MTV & $37.59(55.56)$ & $46.6(37.04)$ & 0.077 & $28.99(50.53)$ & $17.47(17.03)$ & 0.447 & $-17.36(48.35)$ & $-65.64(23.23)$ & 0.0001 \\
\hline \multicolumn{10}{|c|}{ Histogram indices } \\
\hline Skewness & $0.5(0.4)$ & $0.3(0.4)$ & 0.016 & $0.42(0.38)$ & $0.33(0.33)$ & 0.417 & $10.03(171.29)$ & 105.63 (344.67) & 0.545 \\
\hline Kurtosis & $3.0(1.3)$ & $2.7(0.7)$ & 0.048 & $2.98(0.84)$ & $2.73(0.39)$ & 0.37 & $3.50(23.57)$ & 4.56 (15.39) & 0.872 \\
\hline Entropy $_{(\log 2)}$ & $3.7(0.6)$ & $4.0(0.5)$ & 0.144 & $3.23(0.77)$ & $3.09(0.82)$ & 0.56 & $-12.77(17.02)$ & $-23.22(16.71)$ & 0.043 \\
\hline Energy & $0.10(0.04)$ & $0.07(0.03)$ & 0.172 & $0.14(0.08)$ & $0.16(0.09)$ & 0.591 & $54.89(80.49)$ & 112.55 (96.27) & 0.019 \\
\hline \multicolumn{10}{|l|}{ Shape indices } \\
\hline Sphericity & $0.99(0.16)$ & $0.95(0.26)$ & 0.515 & $0.94(0.30)$ & $0.81(0.42)$ & 0.242 & $-5.59(26.35)$ & $-19.55(41.74)$ & 0.389 \\
\hline Compacity & $1.7(0.7)$ & $1.9(0.7)$ & 0.063 & $1.45(0.73)$ & $1.19(0.74)$ & 0.427 & $-15.02(30.21)$ & $-44.58(31.80)$ & 0.0005 \\
\hline \multicolumn{10}{|l|}{ GLCM } \\
\hline Contrast & $0.41(0.09)$ & $0.39(0.08)$ & 0.436 & $0.43(0.16)$ & $0.38(0.22)$ & 0.62 & $8.16(35.71)$ & $-3.09(54.03)$ & 0.783 \\
\hline Correlation & $0.02(0.01)$ & $0.01(0.01)$ & 0.159 & $0.03(0.03)$ & $0.03(0.03)$ & 0.765 & $114.29(238.48)$ & $171.28(216.00)$ & 0.3001 \\
\hline Entropy $_{(\log 2)}$ & $0.55(0.15)$ & $0.60(0.13)$ & 0.277 & $0.44(0.20)$ & $0.40(0.25)$ & 0.713 & $-14.08(54.67)$ & $-33.26(45.40)$ & 0.183 \\
\hline Dissimilarity & $2.02(0.33)$ & $2.17(0.25)$ & 0.118 & $1.65(0.64)$ & $1.41(0.81)$ & 0.301 & $-17.09(27.48)$ & $-36.68(35.00)$ & 0.022 \\
\hline \multicolumn{10}{|l|}{ NGLDM } \\
\hline Coarseness & $524.33(643.33)$ & 704.76 (518.63) & 0.026 & $342.81(487.42)$ & $222.71(227.81)$ & 0.334 & $-28.55(51.36)$ & $-71.44(24.14)$ & 0.0006 \\
\hline Contrast & $0.89(0.05$ & $0.90(0.04)$ & 0.885 & $0.79(0.26)$ & $0.69(0.36)$ & 0.37 & $-8.88(25.71)$ & $-22.59(40.27)$ & 0.123 \\
\hline Busyness & $0.02(0.016)$ & $0.02(0.011)$ & 0.074 & $0.027(0.021)$ & $0.025(0.023)$ & 0.626 & $52.41(143.84)$ & $119.57(178.80)$ & 0.057 \\
\hline
\end{tabular}

${ }^{\star}$ Percentage change calculated by $100 \times[($ value at T2 - value at T1)/value at T1].

MTV = metabolic tumor volume; NGLDM = neighboring gray-level dependence matrix.

Data are mean followed by SD in parentheses. 

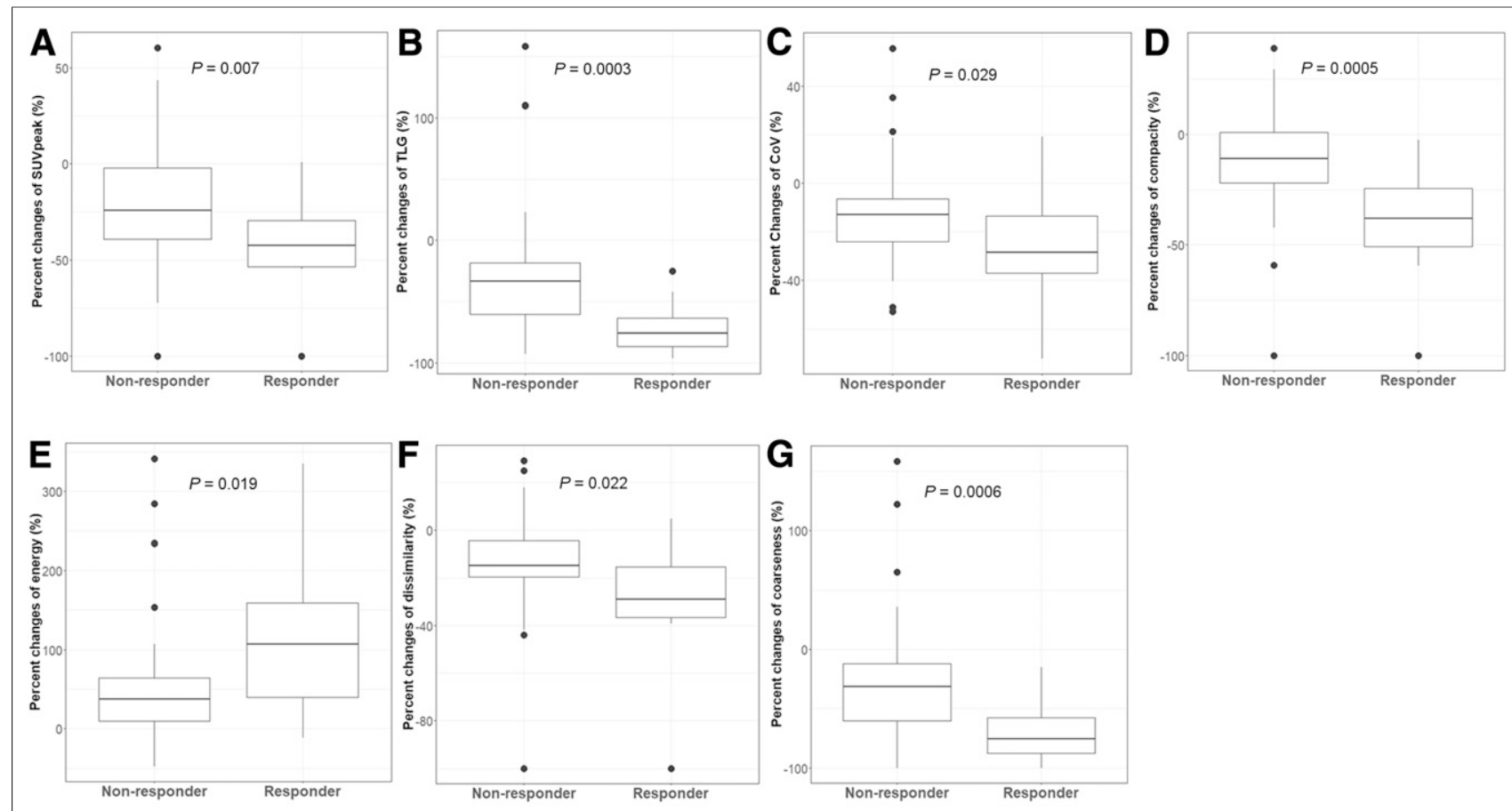

FIGURE 2. Percentage change in metabolic parameters in relation to best response: SUV peak (A), TLG (B), CoV (C), compacity (D), energy (E), dissimilarity $(\mathrm{F})$, and coarseness $(\mathrm{G})$.

Patients' baseline characteristics are listed in Supplemental Table 2. Among 62 patients who were evaluable for tumor response to chemotherapy, 16 had a partial response and were classified as responders and 46 were classified as nonresponders ( 39 with stable disease and 7 with progressive disease). The best objective response rate was $25.8 \%$ (95\% CI, $14.6 \%-37.0 \%$ ), and the disease control rate was $88.7 \%$ (95\% CI, 80.6\%-96.8\%). During the median duration of follow-up (11.2 mo), 55 (88.7\%) patients experienced disease progression and died. The median PFS was 7.1 mo (95\% CI, 5.1-9.7 mo). The median OS was 10.1 mo (95\% CI, 8.6$12.7 \mathrm{mo})$.

\section{Distributions of SUV and TA Parameters at T1 and T2, and Their Changes During First-Line Chemotherapy}

The SUVs and TA parameters at T1 and T2, and changes in values between T1 and T2, are shown in Supplemental Table 3 and Supplemental Figure 2. Except for skewness, kurtosis, sphericity, and contrast GLCM, significant changes between T1 and $\mathrm{T} 2$ occurred for most variables. Overall, the values of all metabolic ITH parameters except busyness were found to decrease with chemotherapy.

\section{Parameters of Responders Versus Nonresponders}

Table 1 compares the SUV and TA parameters of responders with those of nonresponders. At T1, the values of skewness and kurtosis in responders were significantly higher than the values in nonresponders, whereas the value for coarseness was significantly lower in responders than in nonresponders (Supplemental Fig. 3). At T2, no differences were observed between any of the variables assessed for the 2 groups. The percentage change in several parameters, including most of the conventional indices and some of the textural indices, between $\mathrm{T} 1$ and $\mathrm{T} 2$, was significantly associated with response. Metabolic parameters such as $\operatorname{SUV}_{\text {peak }}(P=0.007)$, TLG $(P=0.0003)$,
$\mathrm{CoV}(P=0.029)$, and metabolic tumor volume $(P=0.0001)$ decreased to a greater degree in responders than in nonresponders. Histology entropy $(P=0.043)$, compacity $(P=0.0005)$, dissimilarity $(P=0.022)$, and coarseness $(P=0.0006)$ also decreased more in

TABLE 2

Multivariable Cox Regression Analysis for PFS and OS

\begin{tabular}{|c|c|c|c|}
\hline Parameter & $\mathrm{aHR}^{\star}$ & $95 \% \mathrm{Cl}$ & $P$ \\
\hline \multicolumn{4}{|l|}{ PFS } \\
\hline Best response & 3.29 & $1.54-7.01$ & 0.002 \\
\hline Compacity at T1 & 2.8 & $1.15-6.83$ & 0.023 \\
\hline Percent change of SUV peak & 2.46 & $1.28-4.71$ & 0.007 \\
\hline $\begin{array}{l}\text { Percent change of } \\
\text { entropy GLCM }\end{array}$ & 2.54 & $1.08-6.00$ & 0.033 \\
\hline \multicolumn{4}{|l|}{ OS } \\
\hline Best response & 2.11 & $1.08-4.15$ & 0.029 \\
\hline CA $19-9$ & 1.79 & $1.02-3.14$ & 0.042 \\
\hline $\mathrm{SUV}_{\text {peak }}$ at T1 & 3.31 & $1.37-8.00$ & 0.008 \\
\hline CoV at T2 & 2.02 & $1.03-3.94$ & 0.04 \\
\hline
\end{tabular}

*This backward-selected multivariable Cox regression model included age, sex, initial disease status, treatment, performance status by Eastern Cooperative Oncology Group, CA 19-9, best response, variables at T1 (skewness and compacity for PFS; SUV $_{\text {peak }}$ and compacity for OS), T2 (SUV max , CoV, entropy GLCM,

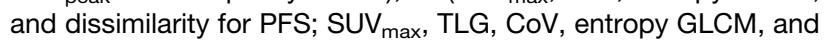
dissimilarity for OS), and, for PFS, percentage changes (SUV $\mathrm{V}_{\text {peak }}$, TLG, CoV, and entropy GLCM).

$\mathrm{aHR}=$ adjusted hazard ratio 




FIGURE 3. Kaplan-Meier survival curves for PFS according to compacity at $T 1(A)$, percentage change in $S_{U} V_{\text {peak }}(B)$, and percentage change in entropy GLCM (C). MPFS = median progression-free survival.

responders than in nonresponders. The value for energy increased 2 -fold in responders over the value in nonresponders $(P=0.019)$. Since energy indicates the uniformity of gray-level voxel pairs, an increased value indicates a decrease in metabolic ITH. Figure 2 shows the decreased metabolic ITH in responders compared with nonresponders.
PFS and OS as Predicted by Clinical Variables and SUV and TA Parameters

The optimal cutoffs and corresponding AUCs for PFS and OS are listed in Supplemental Table 4. By univariate Cox regression analysis, patients with a high level of CA 19-9 and nonresponders had a shorter PFS than those with lower CA 19-9 levels and responders. Significant predictors for PFS among metabolic parameters are listed in Supplemental Table 5. After omission of variables with multicollinearity, the final model for PFS showed that high pretreatment compacity (hazard ratio [HR], 2.8; 95\% CI, 1.15-6.83; $P=0.023$ ), a reduction in $\mathrm{SUV}_{\text {peak }}$ less than $15.45 \%$ (HR, 2.46; 95\% CI, 1.28-4.71; $P=0.007$ ), Entropy GLCM lower than $26.12 \%$ (HR, 2.54; 95\% CI, $1.08-6.00 ; P=0.033$ ), and being a nonresponder (HR, 3.29; 95\% CI, 1.54-7.01; $P=0.02$ ) were independently associated with poor PFS (Table 2; Fig. 3).

An increased pretreatment CA 19-9 level was a significantly poor prognostic factor for OS. Increased $\mathrm{SUV}_{\text {peak }}$, compacity, and dissimilarity at $\mathrm{T} 1$ were associated with shorter OS. At T2, OS was shorter in patients with high $\mathrm{SUV}_{\max }$, TLG, CoV, histologic entropy, compacity, entropy GLCM, dissimilarity, and coarseness (Supplemental Table 5). None of the changes in parameters with an AUC of less than 0.6 were included in the univariate analysis for OS. By multivariable Cox regression analysis, an increased CA 19-9 level (HR, 1.79; 95\% CI, 1.02-3.14; $P=0.042$ ), being a nonresponder (HR, 2.11; 95\% CI, 1.08-4.15; $P=0.02)$, high pretreatment $\mathrm{SUV}_{\text {peak }}(\mathrm{HR}, 3.31 ; 95 \% \mathrm{CI}, 1.37-8.00 ; P=0.008)$, and high $\mathrm{CoV}$ at T2 (HR, 2.02; 95\% CI, 1.03-3.94; $P=0.04$ ) were associated with shorter OS (Table 2; Fig. 4).

\section{DISCUSSION}

Previous studies that have had limitations in design $(9,10)$ have investigated the temporal changes in metabolic ITH and the predictive role of changes in metabolic ITH for survival in patients with advanced pancreatic cancer. We demonstrated that metabolic ITH decreased during palliative chemotherapy to a greater degree in responders than in nonresponders. To our knowledge, this is the first report to demonstrate prospectively the negative changes in ITH over time in patients with advanced pancreatic cancer who were treated with systemic chemotherapy. Furthermore, we found that a lower reduction in heterogeneity, represented as entropy GLCM, during a response assessment period was associated with shortened survival. These findings suggest that in addition to the clinical features of patients with pancreatic cancer, metabolic features of the tumor that are revealed on imaging can predict survival outcomes $(9,10,18)$.

SUVs and most TA parameters were significantly reduced with decreasing metabolic ITH over the course of chemotherapy in our analysis, whereas only 3 parameters $-\mathrm{SUV}_{\text {mean }}, \mathrm{SUV}_{\max }$, and firstorder entropy-decreased in lung cancer patients treated with erlotinib (17). Negative treatment-related trends over time have also been described for patients with other types of tumors $(3,17,18)$. These findings support the hypothesis that tumor clones were initially heterogeneous, and then, as a result of treatment, the predominant subclones disappeared and tumor heterogeneity decreased $(8,19)$.

Greater reductions in metabolic uptake and heterogeneity in the tumors of responders than in the tumors of nonresponders have also been found in other studies, although those studies evaluated localized chemoradiation therapy $(3,18,20)$ or neoadjuvant therapy (5). In addition to the previously reported improvements in SUV $(14,17)$ and TLG $(20,21)$ during treatment, our findings indicate that the gray-level distributions in the tumor become less random and 


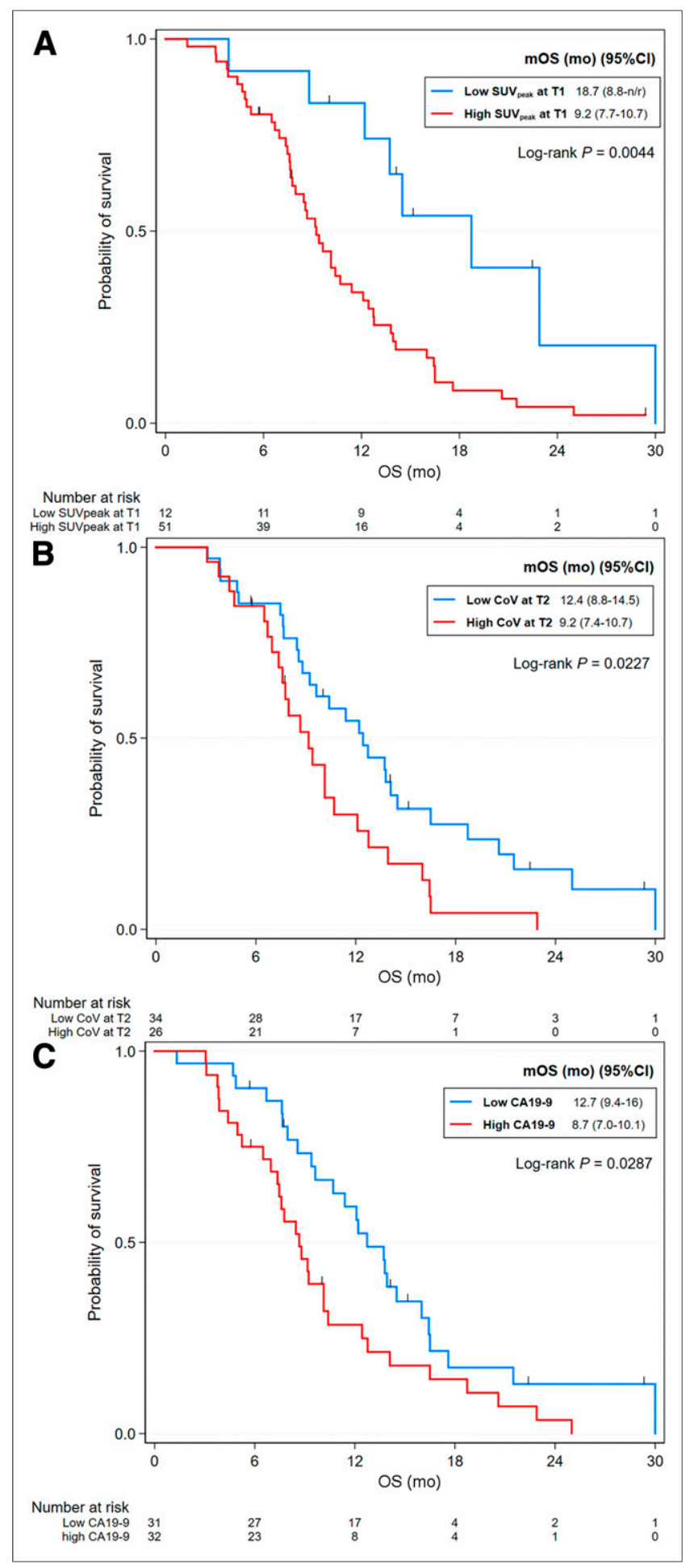

FIGURE 4. Kaplan-Meier survival curves for OS according to SUV peak at T1 (A), CoV at T1 (B), and baseline CA 19-9 level (C). mOS = median overall survival.

more unified than seen at baseline, and the variations in gray-level voxel pairs become minimized. This finding might indicate that an early metabolic response and change in tumor composition precede tumor regression (22).

The reduction in metabolic parameters was also shown to be a significant predictor of PFS. Our finding that the percentage change in $\mathrm{SUV}_{\text {peak }}$ and entropy GLCM was significantly associated with PFS is consistent with the results of other studies (5,10,23-25). An increasing reduction in randomness in gray-level voxel pairs is associated with a lengthening PFS. Although the performance capacity of TA parameters was found in some studies to be superior to that of SUV parameters (5), we found that both SUV and TA parameters predicted PFS and predicted the best response in the multivariable model. We also showed that a highly compact VOI was associated with poor PFS. Despite its clinical significance in our study, whether metabolic compacity is related to such biologic characteristics as tumor density and cellularity has been unclear. Further investigation is warranted to explore such relationships.

We found that both pretreatment CA 19-9 level and SUV peak were important prognostic factors for OS, compared with TAbased parameters. Baseline CA 19-9 level is a well-known independent prognostic factor in pancreatic cancer patients (9). Although pretreatment $\mathrm{SUV}_{\text {peak }}$ is a poor prognostic factor for OS (25), the final model for OS from 2 studies in pancreatic cancer patients did not include it $(9,10)$. A more heterogeneous study population, and treatment modalities different from those of our study, might account for discrepancies. Interestingly, we saw a negative prognostic role for an elevated $\mathrm{CoV}$ at $\mathrm{T} 2$. A high $\mathrm{CoV}$ represents a high degree of heterogeneity; therefore, it might be obvious that a $\mathrm{CoV}$ that remains elevated at the first assessment might be a predictor of poor outcome.

This study had limitations. First, a small number of patients were involved. Larger study cohorts might be needed to confirm our findings. Second, we evaluated the metabolic ITH of only the primary pancreatic lesion, which might not represent the overall metabolic heterogeneity of every tumor lesion in patients with metastatic lesions (18). Third, the heterogeneity of the chemotherapy regimens that our patients received might have confounding effects. However, treatment was an insignificant variable in univariate analyses, and we included it for adjustment in the multivariable models. Finally, this study contained only the training step because of the limited number of participants. The validation step is needed using further cohort data (internal) or independent data (external).

Despite these limitations, examining the association of metabolic ITH with treatment outcomes in patients receiving palliative chemotherapy is warranted. Our study enhanced the significance of metabolic ITH in patients with advanced pancreatic cancer. Although a consensus on the best methodology for assessing metabolic ITH has not yet been achieved, we performed a comprehensive analysis of classic and textural parameters. Finally, we focused on both pretreatment and posttreatment images, whereas most studies have focused on analysis of only the pretreatment one (9). In addition, early $\Delta$-radiomics features in metabolic ITH were good predictors in PFS. In clinical perspectives, one should be cautious in drawing a conclusion that TA parameters can be used for treatment decision making, such as discontinuation of palliative chemotherapy. However, we suggest that TA parameters can be helpful to predict treatment outcomes rather than being important to determine discontinuation of treatment.

\section{CONCLUSION}

Our study demonstrated that the level of metabolic ITH decreases during palliative chemotherapy in patients with advanced pancreatic cancer and that metabolic ITH is associated with response to therapy and is predictive of PFS and OS. 


\section{DISCLOSURE}

This research was supported by the Seoul National University Hospital Research Fund (grant 25-2014-0140) and a grant from the SNU Invitation Program for Distinguished Scholars to DoYoun Oh. The research was also partly supported by a 2018 Seoul National University research grant (800-20180367) and a grant to Gi Jeong Cheon from the Korea Health Technology R\&D Project through the Korea Health Industry Development Institute (KHIDI), which is funded by the Ministry of Health and Welfare, Republic of Korea (grant HI14C1072). No other potential conflict of interest relevant to this article was reported.

\section{KEY POINTS}

QUESTION: Is the temporal change in metabolic ITH during palliative chemotherapy associated with treatment outcomes in patients with advanced pancreatic cancer?

PERTINENT FINDINGS: In a prospective study of 63 patients with advanced pancreatic cancer, we found that metabolic ITH decreased during palliative chemotherapy to a greater degree in responders than in nonresponders. In addition to the clinical variables, a decreased reduction in heterogeneity was associated with shortened PFS and OS.

IMPLICATIONS FOR PATIENT CARE: Measurement of early changes in metabolic ITH during palliative chemotherapy might be an imaging biomarker to predict survival outcome.

\section{REFERENCES}

1. O'Connor JP, Rose CJ, Waterton JC, Carano RA, Parker GJ, Jackson A. Imaging intratumor heterogeneity: role in therapy response, resistance, and clinical outcome. Clin Cancer Res. 2015;21:249-257.

2. Davnall F, Yip CS, Ljungqvist G, et al. Assessment of tumor heterogeneity: an emerging imaging tool for clinical practice? Insights Imaging. 2012;3:573-589.

3. Yang F, Thomas MA, Dehdashti F, Grigsby PW. Temporal analysis of intratumoral metabolic heterogeneity characterized by textural features in cervical cancer. Eur J Nucl Med Mol Imaging. 2013;40:716-727.

4. Kang SR, Song HC, Byun BH, et al. Intratumoral metabolic heterogeneity for prediction of disease progression after concurrent chemoradiotherapy in patients with inoperable stage III non-small-cell lung cancer. Nucl Med Mol Imaging. 2014;48: 16-25.

5. Yip SS, Coroller TP, Sanford NN, Mamon H, Aerts HJ, Berbeco RI. Relationship between the temporal changes in positron-emission-tomography-imaging-based textural features and pathologic response and survival in esophageal cancer patients. Front Oncol. 2016;6:72.

6. Bundschuh RA, Dinges J, Neumann L, et al. Textural parameters of tumor heterogeneity in ${ }^{18} \mathrm{~F}$-FDG PET/CT for therapy response assessment and prognosis in patients with locally advanced rectal cancer. J Nucl Med. 2014;55:891-897.
7. Stathis A, Moore MJ. Advanced pancreatic carcinoma: current treatment and future challenges. Nat Rev Clin Oncol. 2010;7:163-172.

8. Cros J, Raffenne J, Couvelard A, Pote N. Tumor heterogeneity in pancreatic adenocarcinoma. Pathobiology. 2018;85:64-71.

9. Hyun SH, Kim HS, Choi SH, et al. Intratumoral heterogeneity of ${ }^{18} \mathrm{~F}$-FDG uptake predicts survival in patients with pancreatic ductal adenocarcinoma. Eur J Nucl Med Mol Imaging. 2016;43:1461-1468.

10. Yue Y, Osipov A, Fraass B, et al. Identifying prognostic intratumor heterogeneity using pre- and post-radiotherapy ${ }^{18} \mathrm{~F}-\mathrm{FDG}$ PET images for pancreatic cancer patients. J Gastrointest Oncol. 2017;8:127-138.

11. Cui Y, Song J, Pollom E, et al. Quantitative analysis of ${ }^{18} \mathrm{~F}$-fluorodeoxyglucose positron emission tomography identifies novel prognostic imaging biomarkers in locally advanced pancreatic cancer patients treated with stereotactic body radiation therapy. Int J Radiat Oncol Biol Phys. 2016;96:102-109.

12. Eisenhauer EA, Therasse P, Bogaerts J, et al. New response evaluation criteria in solid tumours: revised RECIST guideline (version 1.1). Eur J Cancer. 2009;45: 228-247.

13. Park S, Ha S, Kwon HW, et al. Prospective evaluation of changes in tumor size and tumor metabolism in patients with advanced gastric cancer undergoing chemotherapy: association and clinical implication. J Nucl Med. 2017;58:899904.

14. Jo J, Kwon HW, Park S, Oh DY, Cheon GJ, Bang YJ. Prospective evaluation of the clinical implications of the tumor metabolism and chemotherapy-related changes in advanced biliary tract cancer. J Nucl Med. 2017;58:1255-1261.

15. Nioche C, Orlhac F, Boughdad S, et al. LIFEx: a freeware for radiomic feature calculation in multimodality imaging to accelerate advances in the characterization of tumor heterogeneity. Cancer Res. 2018;78:4786-4789.

16. Bashir U, Siddique MM, McLean E, Goh V, Cook GJ. Imaging heterogeneity in lung cancer: techniques, applications, and challenges. AJR. 2016;207:534-543.

17. Cook GJ, O'Brien ME, Siddique M, et al. Non-small cell lung cancer treated with erlotinib: heterogeneity of ${ }^{18} \mathrm{~F}$-FDG uptake at PET-association with treatment response and prognosis. Radiology. 2015;276:883-893.

18. Dong X, Sun X, Sun L, et al. Early change in metabolic tumor heterogeneity during chemoradiotherapy and its prognostic value for patients with locally advanced non-small cell lung cancer. PLoS One. 2016;11:e0157836.

19. Heeschen C, Sancho P. More challenges ahead-metabolic heterogeneity of pancreatic cancer stem cells. Mol Cell Oncol. 2016;3:e1105353.

20. Usmanij EA, de Geus-Oei LF, Troost EG, et al. ${ }^{18}$ F-FDG PET early response evaluation of locally advanced non-small cell lung cancer treated with concomitant chemoradiotherapy. J Nucl Med. 2013;54:1528-1534.

21. van Helden EJ, Vacher YJL, van Wieringen WN, et al. Radiomics analysis of pre-treatment $\left[{ }^{18} \mathrm{~F}\right] \mathrm{FDG} \mathrm{PET} / \mathrm{CT}$ for patients with metastatic colorectal cancer undergoing palliative systemic treatment. Eur J Nucl Med Mol Imaging. 2018;45: 2307-2317.

22. Van den Abbeele AD. The lessons of GIST-PET and PET/CT: a new paradigm for imaging. Oncologist. 2008;13(suppl 2):8-13.

23. Park $\mathrm{S}$, Ha S, Lee $\mathrm{SH}$, et al. Intratumoral heterogeneity characterized by pretreatment PET in non-small cell lung cancer patients predicts progression-free survival on EGFR tyrosine kinase inhibitor. PLoS One. 2018;13:e0189766.

24. Wu J, Aguilera T, Shultz D, et al. Early-stage non-small cell lung cancer: quantitative imaging characteristics of ${ }^{18} \mathrm{~F}$-fluorodeoxyglucose PET/CT allow prediction of distant metastasis. Radiology. 2016;281:270-278.

25. Vanderhoek M, Perlman SB, Jeraj R. Impact of the definition of peak standardized uptake value on quantification of treatment response. J Nucl Med. 2012;53: $4-11$. 